\title{
SOLUCIÓN LOCAL PARA UNA ECUACIÓN DEL CALOR DEGENERADA NO LINEAL
}

\author{
Teófanes Quispe Méndez ${ }^{1}$
}

Resumen.- En el presente trabajo, estudiamos la existencia de las soluciones locales del problema mixto para un tipo de ecuación del calor degenerada no lineal con una función de Lewis generalizada.

Palabras Claves.- Solución local, Ecuación del calor degenerada no lineal, Método de Galerkin, Método de restimativas de Tartar, Ecuación de evolución.

\section{LOCAL SOLUTION FOR A NONLINEAR DEGENERATE HEAT EQUATION}

\begin{abstract}
In present work, we study the existence of the local solutions to the mixed problem for a type of nonlinear degenerate heat equation with a generalized Lewis function.
\end{abstract}

Key Words.- Local solution, Nonlinear degenerate heat equation, Galerkin method, Tartar estimates method, Evolution equation.

\section{INTRODUCCIÓN}

En este artículo consideramos el siguiente problema de valores iníciales y frontera con una función de Lewis generalizada $\alpha(x, t)$ :

$$
\begin{array}{ll}
\alpha(x, t) u_{t}-\beta(t) \Delta u_{t}-\Delta_{p} u=f(u) & , x \in \Omega, t \geq 0 \\
u=0 & , x \in \partial \Omega, t \geq 0, \\
u(x, 0)=u_{0}(x) & , x \in \Omega,
\end{array}
$$

donde $\Omega$ es un conjunto abierto y acotado de $\mathbb{R}^{n}$ con frontera bien regular $\partial \Omega, \Delta$ es el operador laplaciano, $\alpha(x, t)$ es una función real positiva para $x \in \Omega, t \geq 0, \beta(t)$ es una función real positiva para $t \geq 0, f(s)$ es una función real no lineal para $s \in \mathbb{R}$, y el operador $p$-laplaciano $-\Delta_{p}$ definido para $p \geq 2$ :

$$
-\Delta_{p} u:=-\sum_{i=1}^{n} \frac{\partial}{\partial x_{i}}\left(\left|\frac{\partial u}{\partial x_{i}}\right|^{p-2} \frac{\partial u}{\partial x_{i}}\right) .
$$

\footnotetext{
${ }^{1}$ Profesor de la Facultad de Ciencias Matemáticas de la Universidad Nacional Mayor de San Marcos, e-mail: tquispem@unmsm.edu.pe
} 
Las ecuaciones de tipo $(1,1)$ son utilizadas para modelar diversos fenómenos y procesos en mecánica, física, tecnología, biología y muchas otras áreas. Por ejemplo, describe el proceso de conducción en plasma, filtración de gases y líquidos en medios porosos, reacciones químicas, procesos de crecimiento y migración de poblaciones y entre otros [10].

En el caso $p=2$, la ecuación en $(1,1)$ se reduce a la ecuación clásica del calor, y ha producido sobre ello una literatura bastante impresionante en cuanto al estudio de existencia y no existencia de las soluciones globales, y las propiedades de estas, citamos algunos de ellos $[2,3,9,10,11,12]$. El caso $p>2$, no se tiene muchos resultados conocidos. Tsutsumi [13], obtiene existencia y no existencia de la soluciones globales con los métodos de aproximación de Galerkin y del pozo potencial, cuando $\alpha \equiv 1, \beta \equiv 0$ y $f(u):= \pm u^{\sigma-1}, \sigma \geq 2$. Messaoudi [5], obtiene singularidad en tiempo finito con energía inicial no positiva con el método de concavidad, cuando $\alpha \equiv 1$ y $\beta \equiv 0$. Zhou [14], obtiene singularidad en tiempo finito con energía inicial positiva restringida y con energía inicial no positiva con el método de concavidad, cuando $\beta \equiv 0$.

En este trabajo probaremos la existencia de las soluciones locales del problema $(1,1)$. En la prueba utilizaremos el método de Galerkin [4] y las estimativas de Tartar [12], y las estrategias seguidas por Tsutsumi [13], Quispe Méndez [7, 8] y Gao and Ma [1].

\section{PRELIMINARES}

En esta sección daremos algunas notaciones, conceptos y lemas sin demostración que serán utilizadas en el desarrollo del presente trabajo.

Sea $\Omega$ un conjunto abierto y acotado de $\mathbb{R}^{n}$ con frontera bien regular $\partial \Omega$. Denotamos el producto interno y la norma de $L^{2}(\Omega)$ y $L^{p}(\Omega)$, con $(.,$.$) y |.|_{p}$, respectivamente, para $1 \leq p \leq \infty$. Además $((.,)$.$) y \|$.$\| , denotarán el producto interno y la norma de H_{0}^{1}(\Omega)$, donde $((u, v)):=\int_{\Omega} \nabla u(x) \cdot \nabla v(x) d x$ es la forma de Dirichlet. En el espacio de Sobolev $W_{0}^{1, p}(\Omega)$ usamos la norma

$$
\|u\|_{1, p}:=\left(\sum_{i=1}^{n}\left|\frac{\partial u}{\partial x_{i}}\right|_{p}^{p}\right)^{\frac{1}{p}} .
$$

Sea $X$ un espacio de Banach, $0<T \leq \infty$ y $1 \leq p \leq \infty$. Representamos con $L^{p}(0, T ; X)$ al espacio de Banach de las funciones vectoriales $u:] 0, T[\longrightarrow X$ tales que son medibles 
y $\|u(t)\|_{X} \in L^{p}(0, T)$, con la norma

$$
\begin{gathered}
\|u\|_{L^{p}(0, T ; X)}:=\left(\int_{0}^{T}\|u(t)\|_{X}^{p} d t\right)^{\frac{1}{p}}, 1 \leq p<\infty \\
\|u\|_{L^{\infty}(0, T ; X)}:=\sup _{0<t<T} \operatorname{ess}\|u(t)\|_{X}, p=\infty .
\end{gathered}
$$

Similarmente, cuando $0<T<\infty$, representaremos con $C^{0}([0, T] ; X)$ al espacio de Banach de las funciones continuas $u:[0, T] \longrightarrow X$, con la norma

$$
\|u\|_{C^{0}([0, T] ; X)}:=\sup _{0 \leq t \leq T}\|u(t)\|_{X}
$$

Denotamos $v^{\prime}:=v_{t} \mathrm{y} v(t)(x):=v(x, t)$

Hipótesis. Imponemos sobre las funciones reales $\alpha(x, t), \beta(t)$ y $f(s)$ las siguientes condiciones:

$(H 1) \alpha \in W^{1, \infty}\left(0, \infty ; L^{\infty}(\Omega)\right)$ y $\alpha(x, t) \geq \alpha_{0}>0, \quad \forall(x, t) \in \Omega \times\left[0, \infty\left[, \alpha_{0}\right.\right.$ es una constante.

$(H 2) \beta \in W^{1, \infty}(0, \infty)$ y $\beta(t) \geq \beta_{0}>0, \forall t \geq 0, \beta_{0}$ es una constante.

(H3) $f \in C^{0}(\mathbb{R})$ y existe una constante positiva $C_{0}$ tal que

$$
|f(s)| \leq C_{0}|s|^{\sigma-1}, \forall s \in \mathbb{R}
$$

donde $2 \leq p<\sigma<\frac{p(n+2)}{n}$

Lema 2.1 (Desigualdad de Sobolev-Poincaré [2].) Para cada función $u \in W_{0}^{1, p}(\Omega)$, se tiene

$$
|u|_{r} \leq B_{0}\|u\|_{1, p}
$$

donde $1 \leq r \leq \frac{p n}{n-p}$ si $1 \leq p<n$ y $1 \leq r<\infty$ si $1 \leq n \leq p$. La constante $B_{0}$ depende solamente de $\Omega, n, p$ y $r$.

Lema 2.2 (Desigualdad de Gagliardo-Nirenberg [2].) Para cada función $u \in W_{0}^{1, p}(\Omega)$, $p \geq 1$ y $q \geq 1$, se cumple

$$
|u|_{r} \leq B_{1}\|u\|_{1, p}^{\rho}|u|_{q}^{1-\rho}
$$


donde

$$
\rho:=\left(\frac{1}{q}-\frac{1}{r}\right)\left(\frac{1}{n}-\frac{1}{p}+\frac{1}{q}\right)^{-1}
$$

$y$

(i) $\operatorname{para} p \geq n=1, q \leq r \leq \infty$;

(ii) para $n>1$ y $p<n, q \leq r \leq \frac{n p}{n-p}$ si $q \leq \frac{n p}{n-p}$ y $\frac{n p}{n-p} \leq r \leq q$ si $q \geq \frac{n p}{n-p}$;

(iii) $\operatorname{para} p=n>1, q \leq r<\infty$;

(iv) para $p>n>1, q \leq r \leq \infty$.

La constante $B_{1}$ depende solamente de $n, p, q$ y r.

Lema 2.3 (Desigualdad Generalizada de Gronwall [6].) Sea $f:[0, \infty[\rightarrow[0, \infty[$ continua, $g:] 0, \infty[\rightarrow] 0, \infty[$ continua y no decreciente y sea $C$ una constante positiva, tal que satisface la inecuación diferencial

$$
f(t) \leq C+\int_{0}^{t} g(f(s)) d s, \forall t \in[0, \infty[
$$

Entonces

$$
f(t) \leq G^{-1}\left(T_{*}\right)<\infty, \forall t \in\left[0, T_{*}\right]
$$

para cualquier número fijo $T_{*}<G(\infty)$, donde

$$
G(t):=\int_{C}^{t} \frac{d s}{g(s)}, \quad \forall t \in[C, \infty[
$$

Más aún, si $G(\infty)=\infty$, entonces

$$
f(t) \leq G^{-1}(t), \quad \forall t \in[0, \infty[
$$

Lema 2.4.El operador p-laplaciano $-\Delta_{p}$ es acotado, estrictamente monótono, semicontinuo y coercivo de $W_{0}^{1, p}(\Omega)$ en $W^{-1, q}(\Omega)$,

$$
\left\langle-\Delta_{p} u, v\right\rangle=\int_{\Omega}|\nabla u|^{p-2} \nabla u \cdot \nabla v d x, \forall u, v \in W_{0}^{1, p}(\Omega)
$$


$y$

$$
\left|\left\langle-\Delta_{p} u, v\right\rangle\right| \leq\|u\|_{1, p}^{p-1}\|v\|_{1, p}, \forall u, v \in W_{0}^{1, p}(\Omega)
$$

donde $W^{-1, q}(\Omega)$ es el espacio dual de $W_{0}^{1, p}(\Omega)$ y $p^{-1}+q^{-1}=1$.

Lema 2.5 (Desigualdad de Young ). Sean $1<p, q<\infty$ y $p^{-1}+q^{-1}=1$. Entonces

$$
a b \leq \frac{a^{p}}{p}+\frac{b^{q}}{q}, \forall a, b \geq 0
$$

Lema 2.6 (Aubin-Lions ) [4]. Sean $B_{0}, B_{1}$ y $B$ tres espacios de Banach tales que $B_{0} \hookrightarrow B \hookrightarrow B_{1}, B_{0}$ y $B_{1}$ reflexivos, y $B_{0} \stackrel{c}{\hookrightarrow} B$. Sea

$$
W:=\left\{v \in L^{p_{0}}\left(0, T ; B_{0}\right) ; v^{\prime} \in L^{p_{1}}\left(0, T ; B_{1}\right)\right\}
$$

donde $0<T<\infty, 1<p_{0}, p_{1}<\infty$; con la norma definida por

$$
\|v\|_{W}:=\|v\|_{L^{p_{0}\left(0, T ; B_{0}\right)}}+\left\|v^{\prime}\right\|_{L^{p_{1}\left(0, T ; B_{1}\right)}} .
$$

Entonces $W$ es un espacio de Banach y $W \stackrel{c}{\hookrightarrow} L^{p_{0}}(0, T ; B)$.

Lema 2.7 (Lions) [4]. Sea $Q$ un abierto acotado de $R_{x}^{n} \times R_{t},\left\{g_{\nu}\right\}$ y $g$ funciones de $L^{q}(Q), 1<q<\infty$, tales que

$$
\left\|g_{\nu}\right\|_{L^{q}(Q)} \leq \text { const, } \forall \nu \in \mathbb{N} \quad \text { y } \quad g_{\nu} \rightarrow g \text { c.t.p. en } Q
$$

Entonces

$$
g_{\nu} \rightarrow g \text { en } L^{q}(Q)
$$

Definición 2.9. Una función $u: \Omega \times[0, T] \rightarrow \mathbb{R}$ es llamada solución del problema $(1,1)$ sobre $[0, T]$ si satisface las condiciones $(1,1)_{2}-(1,1)_{3}$ y la igualdad

$$
\alpha(x, t) u_{t}-\beta(t) \Delta u_{t}-\Delta_{p} u=f(u) \quad \text { en } \quad L^{2}\left(0, T ; W^{-1, q}(\Omega)\right)
$$

donde $p^{-1}+q^{-1}=1$. 


\section{EL RESULTADO PRINCIPAL}

En esta sección estudiaremos la solución local del problema $(1,1)$ utilizando los métodos de aproximación de Galerkin [4] y las estimativas de Tartar [12].

Teorema 3.1 (Existencia Local). Supongamos que las funciones $\alpha, \beta$ y $f$ satisfacen las hipótesis $(H 1)-(H 3)$, respectivamente, y que $u_{0} \in W_{0}^{1, p}(\Omega)$. Entonces existe un número positivo $T_{0}$ tal que el problema $(1,1)$ admite una solución u sobre $\left[0, T_{0}\right]$ tal que

$$
u \in L^{\infty}\left(0, T_{0} ; W_{0}^{1, p}(\Omega)\right) \text { y } u^{\prime} \in L^{2}\left(0, T_{0} ; H_{0}^{1}(\Omega)\right)
$$

Demostración. Procedemos en cinco etapas.

Soluciones Aproximadas. Sea $\left\{w_{k}\right\}$ un sistema completo de funciones en $W_{0}^{1, p}(\Omega)$. Sea $V_{m}=\left[w_{1}, w_{2}, \ldots, w_{m}\right]$ el subespacio generado por las primeras $m$ funciones $w_{1}, w_{2}, \ldots, w_{m}$ de $\left\{w_{k}\right\}$.

Sea

$$
u_{m}(t)=\sum_{j=1}^{m} g_{j m}(t) w_{j},
$$

las soluciones aproximadas en $V_{m}$ del problema $(1,1)$, donde las funciones $g_{j m}(t), j=1,2, \ldots, m$, son determinadas del siguiente problema en ecuaciones diferenciales ordinarias, para $w \in V_{m}$

$$
\begin{aligned}
& \left(\alpha(t) u_{m}^{\prime}(t), w\right)+\beta(t)\left(-\Delta u_{m}^{\prime}(t), w\right)+\left(-\Delta_{p} u_{m}(t), w\right)=\left(f\left(u_{m}(t)\right), w\right), \\
& u_{m}(0)=u_{0 m},
\end{aligned}
$$

donde

$$
u_{0 m}=\sum_{j=1}^{m} g_{0 j m} w_{j}, \quad u_{0 m} \rightarrow u_{0} \quad \text { fuerte en } W_{0}^{1, p}(\Omega)
$$

El sistema de ecuaciones ordinarias $(3,2)$ tiene una solución local en el intervalo $\left[0, T_{m}[\right.$. Las siguientes estimativas a priori nos permitirá extender la solución $u_{m}$ a un intervalo $\left[0, T_{0}\right]$ independiente de $m$. 
Estimativa a Priori I. Tomando $w=u_{m}(t)$ en $(3,2)$, obtenemos

$$
\begin{aligned}
\frac{1}{2} \frac{d}{d t}\left|\sqrt{\alpha(t)} u_{m}(t)\right|_{2}^{2}+\frac{1}{2} \beta(t) \frac{d}{d t}\left\|u_{m}(t)\right\|^{2}+\left\|u_{m}(t)\right\|_{1, p}^{p} \\
=\left(f\left(u_{m}(t)\right), u_{m}(t)\right)+\frac{1}{2}\left(\alpha^{\prime}(t) u_{m}(t), u_{m}(t)\right) .
\end{aligned}
$$

De $(3,4)$, resulta

$$
\begin{aligned}
\frac{1}{2} \frac{d}{d t} \varphi_{m}(t)+\left\|u_{m}(t)\right\|_{1, p}^{p}= & \left(f\left(u_{m}(t)\right), u_{m}(t)\right) \\
& +\frac{1}{2}\left(\alpha^{\prime}(t) u_{m}(t), u_{m}(t)\right) \\
& +\frac{1}{2} \beta^{\prime}(t)\left\|u_{m}(t)\right\|^{2}
\end{aligned}
$$

donde

$$
\varphi_{m}(t):=\left|\sqrt{\alpha(t)} u_{m}(t)\right|_{2}^{2}+\beta(t)\left\|u_{m}(t)\right\|^{2} .
$$

Por la hipótesis (H3) y la Desigualdad de Gagliardo-Nirenberg, tenemos

$$
\begin{aligned}
\left|\left(f\left(u_{m}(t)\right), u_{m}(t)\right)\right| & \leq C_{0}\left|u_{m}(t)\right|_{\sigma}^{\sigma} \\
& \leq C_{0} B_{1}^{\sigma}\left\|u_{m}(t)\right\|_{1, p}^{p \delta_{1}}\left|u_{m}(t)\right|_{2}^{2 \delta_{2}}
\end{aligned}
$$

donde

$$
\delta_{1}:=\frac{(\sigma-2) n}{n p+2 p-2 n}<1
$$

y

$$
\delta_{2}:=\frac{\sigma(n p+2 p-2 n)-(\sigma-2) n p}{2(n p+2 p-2 n)}>0 .
$$

Aplicando en $(3,7)$ la Desigualdad de Young, resulta

$$
\left|\left(f\left(u_{m}(t)\right), u_{m}(t)\right)\right| \leq \frac{1}{2 p}\left\|u_{m}(t)\right\|_{1, p}^{p}+\frac{1}{2} C_{1}\left|u_{m}(t)\right|_{2}^{2 \delta_{3}}
$$

donde $\delta_{3}:=\frac{\delta_{2}}{1-\delta_{1}}>1$ y $C_{1}:=2 \frac{\delta_{2}}{\delta_{3}}\left[C_{0} B_{1}^{\sigma}\left(2 p \delta_{1}\right)^{\delta_{1}}\right]^{\frac{\delta_{3}}{\delta_{2}}}$.

Por las hipótesis $(H 1)-(H 2)$, se tiene $\alpha^{\prime}(x, t) \leq\left\|\alpha^{\prime}\right\|_{L^{\infty}\left(0, \infty ; L^{\infty}(\Omega)\right)}$ c.t.p. para $(x, t) \in \Omega \times\left[0, \infty\left[\right.\right.$ y $\beta^{\prime}(t) \leq\left\|\beta^{\prime}\right\|_{L^{\infty}(0, \infty)}$ c.t.p. para $t \in[0, \infty[$. Tenemos

$$
\left(\alpha^{\prime}(t) u_{m}(t), u_{m}(t)\right)+\beta^{\prime}(t)\left\|u_{m}(t)\right\|^{2} \leq C_{\alpha \beta}\left[\left|u_{m}(t)\right|_{2}^{2}+\left\|u_{m}(t)\right\|^{2}\right]
$$

donde $C_{\alpha \beta}:=\operatorname{máx}\left\{\left\|\alpha^{\prime}\right\|_{L^{\infty}\left(0, \infty ; L^{\infty}(\Omega)\right)},\left\|\beta^{\prime}\right\|_{L^{\infty}(0, \infty)}\right\}$. 
Por los resultados $(3,8)$ y $(3,9)$, de $(3,5)$ conseguimos

$$
\frac{d}{d t} \varphi_{m}(t) \leq C_{2}\left[\left|u_{m}(t)\right|_{2}^{2 \delta_{3}}+\left|u_{m}(t)\right|_{2}^{2}+\left\|u_{m}(t)\right\|^{2}\right]
$$

donde $C_{2}:=\operatorname{máx}\left\{C_{1}, C_{\alpha \beta}\right\}$. También por $(H 1)-(H 2)$ y $(3,3)$, resultan

$$
\left|u_{m}(t)\right|_{2}^{2}+\left\|u_{m}(t)\right\|^{2} \leq \frac{1}{\min \left\{\alpha_{0}, \beta_{0}\right\}} \varphi_{m}(t)
$$

y

$$
\left|\sqrt{\alpha(0)} u_{0 m}\right|_{2}^{2}+\beta(0)\left\|u_{0 m}\right\|^{2}=\varphi_{m}(0) \leq C_{3}
$$

donde $C_{3}$ es una constante positiva independiente de $m$.

Por $(3,11)-(3,12)$, integrando $(3,10)$ sobre $[0, t]$, obtenemos

$$
\psi_{m}(t) \leq C+K \int_{0}^{t}\left[\psi_{m}^{\delta_{3}}(s)+\psi_{m}(s)\right] \cdot d s
$$

donde

$$
\begin{gathered}
\psi_{m}(t):=\left|u_{m}(t)\right|_{2}^{2}+\left\|u_{m}(t)\right\|^{2} \\
C:=\frac{C_{3}}{\min \left\{\alpha_{0}, \beta_{0}\right\}} \text { y } K:=\frac{C_{2}}{\min \left\{\alpha_{0}, \beta_{0}\right\}} .
\end{gathered}
$$

Consideremos las funciones

$$
g(s):=K\left(s^{\delta_{3}}+s\right) \quad \text { y } \quad G(t):=\int_{C}^{t} \frac{d s}{g(s)}
$$

Observemos que la función $g(s)$ es estrictamente creciente y cóncava hacia arriba para todo $s \geq 0$, por ser $\delta_{3}>1$. Desde que $\frac{1}{g(s)} \leq \frac{1}{K s^{\delta_{3}}}, \forall s \geq C$, resulta

$$
G(\infty) \leq \frac{1}{K\left(\delta_{3}-1\right) C^{\delta_{3}-1}}
$$

Por $(3,14)$ y la Desigualdad Generalizada de Gronwall, de $(3,13)$ existe un número $T_{0}>0$ y una constante positiva $C_{4}$ independientes de $m$ tal que

$$
\left|u_{m}(t)\right|_{2}^{2}+\left\|u_{m}(t)\right\|^{2} \leq C_{4}, \quad \forall t \in\left[0, T_{0}\right]
$$

Observemos que $0<T_{0}<G(\infty)$ y $C_{4}:=G^{-1}\left(T_{0}\right)$ 
Estimativa a Priori II. Tomando $w=u_{m}^{\prime}(t)$ en $(3,2)$, obtenemos

$$
\frac{d}{d t} E(t)+\left|\sqrt{\alpha(t)} u_{m}^{\prime}(t)\right|_{2}^{2}+\beta(t)\left\|u_{m}^{\prime}(t)\right\|^{2}=0
$$

donde

$$
E(t):=\frac{1}{p}\left\|u_{m}(t)\right\|_{1, p}^{p}-\int_{\Omega} F\left(u_{m}(x, t)\right) d x
$$

y

$$
F(s):=\int_{0}^{s} f(\xi) d \xi
$$

Integrando $(3,16)$ sobre $[0, t]$, resulta

$$
E(t)+\int_{0}^{t}\left|\sqrt{\alpha(s)} u_{m}^{\prime}(s)\right|_{2}^{2} d s+\int_{0}^{t} \beta(s)\left\|u_{m}^{\prime}(t)\right\|^{2} d s=E(0)
$$

donde

$$
E(0):=\frac{1}{p}\left\|u_{0 m}\right\|_{1, p}^{p}-\int_{\Omega} F\left(u_{0 m}(x)\right) d x
$$

Por $(3,3)$, se obtiene

$$
E(0) \leq C_{5}
$$

donde $C_{5}$ es una constante positiva independiente de $m$. De $(3,17)$, obtenemos

$$
\begin{aligned}
\frac{1}{p}\left\|u_{m}(t)\right\|_{1, p}^{p}+ & \int_{0}^{t}\left|\sqrt{\alpha(s)} u_{m}^{\prime}(s)\right|_{2}^{2} d s \\
& +\int_{0}^{t} \beta(s)\left\|u_{m}^{\prime}(t)\right\|^{2} d s \leq C_{5}+\int_{\Omega} F\left(u_{m}(x, t)\right) d x .
\end{aligned}
$$

Por la hipótesis (H3), y las Desigualdades de Gagliardo-Nirenberg y Young, resulta

$$
\begin{aligned}
\int_{\Omega}\left|F\left(u_{m}(x, t)\right)\right| d x & \leq \frac{C_{0}}{\sigma}\left|u_{m}(t)\right|_{\sigma}^{\sigma} \\
& \leq \frac{1}{2 p}\left\|u_{m}(t)\right\|_{1, p}^{p}+C_{6}\left|u_{m}(t)\right|_{2}^{2 \delta_{3}}
\end{aligned}
$$

donde $\delta_{3}:=\frac{\delta_{2}}{1-\delta_{1}}>1$ y $C_{6}:=\frac{\delta_{2}}{\delta_{3}}\left[\frac{C_{0}}{\sigma} B_{1}^{\sigma}\left(2 p \delta_{1}\right)^{\delta_{1}}\right]^{\frac{\delta_{3}}{\delta_{2}}}$. Por hipótesis $(H 1)-(H 2),(3,15) \mathrm{y}$ $(3,19)$, de $(3,18)$ se obtiene

$$
\left\|u_{m}(t)\right\|_{1, p}^{p}+\int_{0}^{t}\left|u_{m}^{\prime}(s)\right|_{2}^{2} d s+\int_{0}^{t}\left\|u_{m}^{\prime}(t)\right\|^{2} d s \leq C_{7}, \quad \forall t \in\left[0, T_{0}\right]
$$

donde $C_{7}$ es una constante positiva independiente de $m$. 
Por el Lema 2.5 y $(3,20)$, resulta

$$
\left\|-\Delta_{p} u_{m}(t)\right\|_{-1, q} \leq C_{8}, \quad \forall t \in\left[0, T_{0}\right]
$$

donde $p^{-1}+q^{-1}=1$ y $C_{8}$ es una constante positiva independiente de $m$.

Pasaje al Límite. De las estimativas $(3,15),(3,20)$ y $(3,21)$, tenemos que

$$
\begin{gathered}
\left\{u_{m}\right\} \text { es acotada en } L^{\infty}\left(0, T_{0} ; W_{0}^{1, p}(\Omega)\right), \\
\left\{u_{m}\right\} \text { es acotada en } L^{\infty}\left(0, T_{0} ; H_{0}^{1}(\Omega)\right), \\
\left\{u_{m}\right\} \text { es acotada en } L^{\infty}\left(0, T_{0} ; L^{2}(\Omega)\right), \\
\left\{u_{m}^{\prime}\right\} \text { es acotada en } L^{2}\left(0, T_{0} ; L^{2}(\Omega)\right) \\
\left\{u_{m}^{\prime}\right\} \text { es acotada en } L^{2}\left(0, T_{0} ; H_{0}^{1}(\Omega)\right) \\
\left\{-\Delta_{p} u_{m}\right\} \text { es acotada en } L^{\infty}\left(0, T_{0} ; W^{-1, q}(\Omega)\right)
\end{gathered}
$$

donde $p^{-1}+q^{-1}=1$. Por $(3,22)-(3,27)$, existen subsucesiones $\left\{u_{\nu}\right\}$ y $\left\{u_{\nu}^{\prime}\right\}$ de $\left\{u_{m}\right\}$ y $\left\{u_{m}^{\prime}\right\}$, respectivamente, tales que

$$
\begin{gathered}
u_{\nu} \stackrel{*}{\rightarrow} u \quad \text { en } L^{\infty}\left(0, T_{0} ; W_{0}^{1, p}(\Omega)\right), \\
u_{\nu} \stackrel{*}{\rightarrow} u \quad \text { en } L^{\infty}\left(0, T_{0} ; H_{0}^{1}(\Omega)\right), \\
u_{\nu} \stackrel{*}{\rightarrow} u \text { en } L^{\infty}\left(0, T_{0} ; L^{2}(\Omega)\right) \\
u_{\nu}^{\prime} \rightarrow u^{\prime} \text { en } L^{2}\left(0, T_{0} ; L^{2}(\Omega)\right) \\
u_{\nu}^{\prime} \rightarrow u^{\prime} \text { en } L^{2}\left(0, T_{0} ; H_{0}^{1}(\Omega)\right) \\
-\Delta_{p} u_{\nu} \stackrel{*}{\rightarrow} \chi \quad \text { en } L^{\infty}\left(0, T_{0} ; W^{-1, q}(\Omega)\right) .
\end{gathered}
$$

Desde que $L^{\infty}\left(0, T_{0} ; H_{0}^{1}(\Omega)\right) \hookrightarrow L^{2}\left(0, T_{0} ; H_{0}^{1}(\Omega)\right)$ y $H_{0}^{1}(\Omega) \stackrel{c}{\hookrightarrow} L^{2}(\Omega)$, aplicando el Lema de Lions-Aubin, de $(3,29)$ y $(3,31)$ resultan

$$
u_{\nu} \rightarrow u \text { fuerte en } L^{2}\left(0, T_{0} ; L^{2}(\Omega)\right)
$$


y

$$
u_{\nu} \rightarrow u \text { c.t.p. en } \Omega \times\left[0, T_{0}\right] \text {. }
$$

Usando la hipótesis $(H 3),(3,22),(3,24)$ y $(3,35)$ vemos que

$$
\begin{aligned}
\int_{0}^{T_{0}} \int_{\Omega}\left|f\left(u_{\nu}(x, t)\right)\right|^{\frac{\sigma}{\sigma-1}} d x d t & \leq \int_{0}^{T_{0}} C_{0}\left|u_{\nu}(t)\right|_{\sigma}^{\sigma} d t \\
& \leq \frac{1}{2} \int_{0}^{T_{0}}\left[\frac{1}{p}\left\|u_{\nu}(t)\right\|_{1, p}^{p}+C_{1}\left|u_{\nu}(t)\right|_{2}^{2 \delta_{3}}\right] d t \\
& \leq C_{9}
\end{aligned}
$$

y

$$
f\left(u_{\nu}\right) \rightarrow f(u) \text { c.t.p. en } \Omega \times\left[0, T_{0}\right]
$$

Por $(3,36)-(3,37)$ y el Lema de Lions, se deduce que

$$
f\left(u_{\nu}\right) \rightarrow f(u) \quad \text { en } \quad L^{\frac{\sigma}{\sigma-1}}\left(0, T_{0} ; L^{\frac{\sigma}{\sigma-1}}(\Omega)\right)
$$

De las convergencias $(3,28)$ - $(3,33)$ y $(3,38)$ por pasaje al límite en la ecuación aproximada $(3,2)$, resulta

$$
\int_{0}^{T_{0}}\left(\alpha(t) u_{t}-\beta(t) \Delta u_{t}+\chi-f(u), v\right) d t=0
$$

para cada $v \in L^{2}\left(0, T_{0} ; W_{0}^{1, p}(\Omega)\right)$, es decir

$$
\alpha(x, t) u_{t}-\beta(t) \Delta u_{t}+\chi=f(u) \text { en } L^{2}\left(0, T_{0} ; W^{-1, q}(\Omega)\right)
$$

donde $p^{-1}+q^{-1}=1$.

Probemos que $\chi=-\Delta_{p} u$. Sea $A:=-\Delta_{p}$. Tomando $w=u_{\nu}(t)$ en $(3,2)$ e integrando 
sobre $[0, t]$, obtenemos

$$
\begin{aligned}
\int_{0}^{t}\left(A u_{\nu}(s), u_{\nu}(s)\right) d s= & \int_{0}^{t}\left(f\left(u_{\nu}(s)\right), u_{\nu}(s)\right) d s \\
& -\frac{1}{2}\left|\sqrt{\alpha(t)} u_{\nu}(t)\right|_{2}^{2}-\frac{1}{2} \beta(t)\left\|u_{\nu}(t)\right\|^{2} \\
& +\frac{1}{2}\left|\sqrt{\alpha(0)} u_{0 \nu}\right|_{2}^{2}+\frac{1}{2} \beta(0)\left\|u_{0 \nu}\right\|^{2} \\
& +\frac{1}{2} \int_{0}^{t}\left(\alpha^{\prime}(s) u_{\nu}(s), u_{\nu}(s)\right) d s \\
& +\frac{1}{2} \int_{0}^{t} \beta^{\prime}(s)\left\|u_{\nu}(s)\right\|^{2} d s .
\end{aligned}
$$

Por $W_{0}^{1, p}(\Omega) \hookrightarrow L^{\sigma}(\Omega),(3,28)$ y $(3,38)$, se logran

$$
u_{\nu} \rightarrow u \text { en } L^{\sigma}(\Omega) \text { c.t. p. para } t \in\left[0, T_{0}\right]
$$

y

$$
\left(f\left(u_{\nu}(t)\right), u_{\nu}(t)\right) \rightarrow(f(u(t)), u(t)) \text { c. t. p. para } t \in\left[0, T_{0}\right] .
$$

Por $(H 3),(3,22)$ y $(3,24)$, resulta

$$
\begin{aligned}
\left|\left(f\left(u_{\nu}(t)\right), u_{\nu}(t)\right)\right| & \leq C_{0}\left|u_{\nu}(t)\right|_{\sigma}^{\sigma} \\
& \leq \frac{1}{2 p}\left\|u_{\nu}(t)\right\|_{1, p}^{p}+\frac{1}{2} C_{1}\left|u_{\nu}(t)\right|_{2}^{2 \delta_{3}} \\
& \leq C_{10}, \text { para } t \in\left[0, T_{0}\right] .
\end{aligned}
$$

De $(3,41),(3,42)$, y el Teorema de Convergencia Dominada de Lebesgue, resulta

$$
\int_{0}^{t}\left(f\left(u_{\nu}(s)\right), u_{\nu}(s)\right) d s \rightarrow \int_{0}^{t}(f(u(s)), u(s)) d s, \text { para } t \in\left[0, T_{0}\right]
$$


Por pasaje al límite en $(3,40)$, haciendo uso de $(3,43)$ y $(3,39)$, obtenemos

$$
\begin{aligned}
\lim _{\nu \rightarrow \infty} \sup \int_{0}^{t}\left(A u_{\nu}(s), u_{\nu}(s)\right) d s \leq & \int_{0}^{t} \lim _{\nu \rightarrow \infty} \sup \left(A u_{\nu}(s), u_{\nu}(s)\right) d s \\
= & \int_{0}^{t}(f(u(s)), u(s)) d s \\
& -\frac{1}{2}|\sqrt{\alpha(t)} u(t)|_{2}^{2}-\frac{1}{2} \beta(t)\|u(t)\|^{2} \\
& +\frac{1}{2}\left|\sqrt{\alpha(0)} u_{0}\right|_{2}^{2}+\frac{1}{2} \beta(0)\left\|u_{0}\right\|^{2} \\
& +\frac{1}{2} \int_{0}^{t}\left(\alpha^{\prime}(s) u(s), u(s)\right) d s \\
& +\frac{1}{2} \int_{0}^{t} \beta^{\prime}(s)\|u(s)\|^{2} d s \\
= & \int_{0}^{t}(\chi(s), u(s)) d s .
\end{aligned}
$$

Para cada $v \in L^{2}\left(0, T_{0} ; W_{0}^{1, p}(\Omega)\right)$, definamos la función

$$
\varphi_{\nu}(t):=\int_{0}^{t}\left(A u_{\nu}(s)-A v(s), u_{\nu}(s)-v(s)\right) d s, \text { para } t \in\left[0, T_{0}\right]
$$

Por la monotonía del operador $A, \varphi_{\nu}(t) \geq 0, \forall t \in\left[0, T_{0}\right]$. Por $(3,44),(3,28)$ y $(3,33)$, resulta

$$
\begin{aligned}
0 & \leq \lim _{\nu \rightarrow \infty} \sup \varphi_{\nu}(t) \\
& \leq \int_{0}^{t} \lim _{\nu \rightarrow \infty} \sup \left(A u_{\nu}(s)-A v(s), u_{\nu}(s)-v(s)\right) d s \\
& \leq \int_{0}^{t}(\chi(s)-A v(s), u(s)-v(s)) d s
\end{aligned}
$$

Tomando $v=u-\lambda w$ en $(3,45)$, donde $\lambda>0$ y $w \in L^{2}\left(0, T_{0} ; W_{0}^{1, p}(\Omega)\right)$, se tiene

$$
\int_{0}^{t}(\chi(s)-A(u(s)-\lambda w(s)), w(s)) d s \geq 0
$$

Desde que $A$ es un operador semicontinuo y haciendo tender $\lambda \rightarrow 0$, logramos

$$
\int_{0}^{t}(\chi(s)-A u(s), w(s)) d s \geq 0, \forall w \in L^{2}\left(0, T_{0} ; W_{0}^{1, p}(\Omega)\right) .
$$

De aqui resulta $\chi=A u$.

El dato inicial se verifica de modo estándar. Con todo esto concluye la demostración 
del Teorema 3.1.

Usando argumentos similares que en la prueba del Teorema 3.1, se obtienen los siguientes dos teoremas.

Teorema 3.2. Supongamos que las funciones a y f satisfacen las hipótesis (H1) y (H3), respectivamente, $\beta \equiv 0$ y $u_{0} \in W_{0}^{1, p}(\Omega)$. Entonces existe un número positivo $T_{0}$ tal que el problema $(1,1)$ admite una solución u sobre $\left[0, T_{0}\right]$ tal que

$$
u \in L^{\infty}\left(0, T_{0} ; W_{0}^{1, p}(\Omega)\right) \text { y } u^{\prime} \in L^{2}\left(0, T_{0} ; L^{2}(\Omega)\right)
$$

Teorema 3.3. Supongamos que las funciones $\beta$ y $f$ satisfacen las hipótesis $(H 2)$ y (H3), respectivamente, $\alpha \equiv 0$ y $u_{0} \in W_{0}^{1, p}(\Omega)$. Entonces existe un número positivo $T_{0}$ tal que el problema $(1,1)$ admite una solución u sobre $\left[0, T_{0}\right]$ tal que

$$
u \in L^{\infty}\left(0, T_{0} ; W_{0}^{1, p}(\Omega)\right) \text { y } u^{\prime} \in L^{2}\left(0, T_{0} ; H_{0}^{1}(\Omega)\right)
$$




\section{REFERENCIAS}

[1] Gao, H. and Ma, T. F. - Global solutions for a nonlinear wave equation with the p-laplacian operator, Electronic Journal of Qualitative Theory of Differential Equations, 11 (1999), pp. 1-13.

[2] Ladyzhenskaya, O. A., Solonnikov, V. A. and Uralceva, N. N. - Linear and Quasilinear Parabolic Equations, "Nauka", Moscow, 1967; English transl., Trans. Math. Monographs, 23, American Mathematical Society, Providence, Rhode Island, 1968.

[3] Levine, H. A. - Some nonexistence and instability theorems for solutions of formally parabolic equations of the form $P u_{t}=-A u+F(u)$, Arch. Rational Mech. Anal., 51 (1973), pp. 371-386.

[4] Lions, J. L. - Quelques méthodes de résolution des problèmes aux limites non linéaires, Dunod, Paris (1969).

[5] Messaoudi, S. A. - A note on blow up of solutions of a quasilinear heat equation with vanishing initial energy, Math. Nachr., J. Math. Anal. Appl. 273 (2002), pp. 243-247.

[6] Nishihara, K. - On a global solution of some quasilinear hyperbolic equation, Tokio J. Math., 7 (1984) 2, pp. 437-459.

[7] Quispe Méndez, T. - Singularidad en tiempo finito para un sistema de Kirchhoff, Tesis de Maestría, Universidad Nacional Mayor de San Marcos, Lima-Perú (1998).

[8] Quispe Méndez, T. - Solución local y singularidad para un sistema de Kirchhoff no lineal, Pesquimat Revista de la Fac. CC. MM. de la Universidad Nacional Mayor de San Marcos Vol. VIII, No.2, pág. 45-62, Lima-Perú. Diciembre 2005.

[9] Rincon, M. A., Limaco, J. and Liu, I-S. - Existence and uniqueness of solutions of a nonlinear heat equation, Tend. Mat. Apl. Comput., 6 (2005) (2), pp. 273-284.

[10] Samarskii, A.A., Galaktionov, V.A., Kurdyumov, S.P. y Mikhailov, A.P. - Blow-up in problems for quasilinear parabolic equations. Nauka, Moscow, 1987 (in Russian). English transl.: Walter de Gruyter, Berlin, 1995.

[11] Tan, Z. - The reaction-diffusion equation with Lewis function and critical Sobolev exponent, J. Math. Anal. Appl., 272 (2002) (2), pp. 480-495.

[12] Tartar, L. - Topics in nonlinear analysis, Publications Mathématiques D'Orsay, Université de Paris-Sud, Orsay 1978.

[13] Tsutsumi, M. - Existence and nonexistence of global solutions for nonsilinear parabolic equations, Publ. RIMS, Kyoto Univ., 8 (1972/73), pp. 211-229.

[14] Zhou, Y. - Global nonexistence for a quasilinear evolution equation with a generalized Lewis function, J. Anal. Appl., 24 (2005) (1), pp. 179-187. 